\title{
FUNCIONALIDADE E QUALIDADE DE VIDA EM IDOSOS SUBMETIDOS A ARTROPLASTIA TOTAL DO JOELHO
}

\author{
FUNCTIONALITY AND QUALITY OF LIFE IN ELDERLY PEOPLE SUBMITTED TO TOTAL KNEE ARTHROPLASTY \\ FUNCIONALIDAD Y CALIDAD DE VIDA EN ANCIANOS SOMETIDOS A ARTROPLASTIA TOTAL DE RODILLA
}

DOI 10.33194/rper.2019.v2.n2.4584 | Submetido 03.09.2019 | Aprovado 01.12.2019

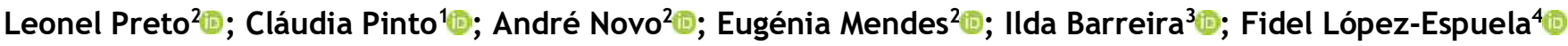 \\ 1 - Hospital Terra Quente; 2 - Instituto Politécnico de Bragança; 3 - Unidade Local de Saúde do Nordeste; \\ 4 - Universidad de Extremadura.
}

\section{RESUMO}

Objetivo: Avaliar a perceção da funcionalidade do joelho e a qualidade de vida relacionada à saúde (QVRS) em idosos submetidos a artroplastia total do joelho (ATJ).

Métodos: Estudaram-se 40 pacientes em dois momentos de avaliação: no pré-operatório e oito semanas após cirurgia. A QVRS foi avaliada através do MOS SF-36 v2 (Medical Outcomes Study 36-Item Short-Form Health Survey) e a perceção da funcionalidade do joelho através do KOOS (Knee injury and Osteoarthritis Outcome Score).

Resultados: Amostra (71,6 68,1 anos) maioritariamente feminina $(62,5 \%)$. Os resultados do MOS SF-36 v2 revelam uma melhoria em todas as dimensões da saúde física na segunda avaliação $(p<0,05)$. No domínio da saúde mental também houve melhoria em todas as dimensões, à exceção da vitalidade. A funcionalidade do joelho melhorou nas subescalas: sintomas $(p=0,015)$, dor $(p<0,001)$ e qualidade de vida $(p<0,001)$.

Conclusão: Pacientes avaliados oito semanas após ATJ revelaram melhorias na saúde autopercebida, qualidade de vida, desempenho físico e dor.

Descritores: Qualidade de Vida; Osteoartrite do Joelho; Artroplastia do Joelho.

\section{RESUMEN}

Objetivo: Evaluar la percepción de la funcionalidad de la rodilla y la calidad de vida relacionada con la salud (CVRS) en ancianos sometidos a artroplastia total de rodilla (ATR).

Métodos: Se estudiaron 40 pacientes en dos momentos de evaluación: en el preoperatorio y ocho semanas después de la cirugía. La CVRS se evaluó utilizando el MOS-SF-36 v2 (Medical Outcomes Study 36-Item Short-Form Health Survey) y la percepción de la funcionalidad de la rodilla a través del KOOS (Knee injury and Osteoarthritis Outcome Score).

Resultados: Muestra $(71,6 \pm 8,1$ años) en su mayoría mujeres $(62,5 \%)$. Los resultados del MOS SF-36 v2 sugieren mejoras en todas las dimensiones de la salud física en la segunda evaluación $(\mathrm{p}<0.05)$. En la salud mental también ha habido mejoras en todas las dimensiones, excepto la vitalidad. La funcionalidad de la rodilla mejoró en las subescalas: síntomas $(p=0.015)$, dolor $(p<0.001)$ y calidad de vida $(p<0.001)$.

Conclusión: Los pacientes evaluados ocho semanas después de ATR revelaron mejoras en la salud percibida, la calidad de vida, el rendimiento físico y el dolor.

Descriptores: Calidad de vida; Osteoartritis de la Rodilla; Artroplastia de Reemplazo de Rodilla.

\section{ABSTRACT}

Objective: To evaluate perception of knee functionality and health-related quality of life (HRQL) in elderly patients submitted to total knee arthroplasty (TKA).

Methods: 40 patients were studied at two moments of evaluation: in the preoperative period and eight weeks after surgery. HRQL was assessed using the MOS-SF-36 V2 (Medical Outcomes Study 36-Item Short-Form Health Survey) and KOOS (Knee Injury and Osteoarthritis Outcome Score).

Results: Sample $(71.6 \pm 8.1$ years), mostly female $(62.5 \%)$. The SF-36 v2 MOS results show an improvement in all dimensions of physical health in the second evaluation $(p<0.05)$. In the field of mental health there has also been improvement in all dimensions, except vitality. The functionality of the knee improved in the subscales: symptoms $(p=0.015)$, pain $(p<0.001)$ and quality of life $(p<0.001)$. 
Conclusion: Patients evaluated eight weeks after TKA showed improvements in self-perceived health, quality of life, physical performance and pain.

Keywords: Quality of Life; Osteoarthritis Knee; Arthroplasty Replacement Knee.

\section{INTRODUÇ̃̃O}

A osteoartrose $(\mathrm{AO})$ é definida como uma condição clínica degenerativa caracterizada pela perda progressiva da cartilagem articular podendo afetar o osso subcondral e comprometer toda a articulação ${ }^{(1)}$. A OA constitui uma das doenças crónicas mais frequentes da atualidade prevendo-se que a sua incidência continue a aumentar em simultâneo com o aumento da esperança média de vida. Ela constituiu a doença articular mais comum em todo o mundo e uma das principais causas de incapacidade crónica, sobretudo na população idosa ${ }^{(2)}$. A idade é o principal fator de risco para o desenvolvimento de OA já que as mudanças que ocorrem a nível celular e tecidular durante o processo de envelhecimento tornam as articulações mais suscetíveis ao dano e incapazes de manter a homeostasia ${ }^{(3)}$.

Sendo a maior e mais solicitada articulação de carga do corpo humano, o joelho é responsável pela maioria das situações de OA, chegando a afetar sintomaticamente $45 \%$ das pessoas idosas ${ }^{(4)}$. Como articulação de carga, o joelho é bastante suscetível às alterações do envelhecimento que potenciam o desenvolvimento da artrose, das quais podemos salientar: as alterações na proprioceção e equilíbrio, a sarcopenia e o aumento de massa gorda, a osteoporose, a degeneração meniscal e a menor hidratação articular ${ }^{(3)}$.

A nível sintomático a OA do joelho origina dor e rigidez articular, edema, deformidade progressiva em varo ou valgo e marcha lenta e claudicante $(3,4)$. As limitações na deambulação, na subida e descida de escadas e no agachar interferem gravemente nas atividades de vida e de lazer. Para além disso prejudica outros aspetos da vida das pessoas idosas, como a interação social, o funcionamento físico e mental e a qualidade do sono ${ }^{(5)}$. De um modo geral, os problemas músculo-esqueléticos interferem na qualidade de vida (QV), podendo constituir-se como causas de invalidez precoce ou ausência ao trabalho por doença ${ }^{(6)}$.

Os sintomas e as limitações provocadas pela patologia têm um significativo impacto na qualidade de vida relacionada à saúde (QVRS) ${ }^{(5)}$, pelo que se torna fundamental o alívio da dor e o controlo dos sintomas pelo tratamento médico ou cirúrgico, variando este de acordo com o grau da doença, o nível de incapacidade, profissão, idade, entre outros fatores.

Para além da artroscopia, osteotomia e artroplastia parcial, o tratamento cirúrgico para OA do joelho inclui a artroplastia total do joelho (ATJ). Nas pessoas idosas sem atividade laboral, e com OA avançada, esta última alternativa constituiu o tratamento mais adequado, económico e seguro ${ }^{(7)}$.

A ATJ tem como objetivos principais diminuir as queixas dolorosas, melhorar a amplitude articular e a capacidade de marcha, permitindo que os pacientes adquiram mais precocemente a sua mobilidade de forma autónoma e melhorem a QV. Assim, após cirurgia, é importante quantificar esses ganhos em saúde através da investigação, quer analisando a evidência científica disponível, quer realizando novos estudos.

Um estudo realizado com 52 mulheres idosas entrevistadas aos 3 e 6 meses após ATJ concluiu que em ambas as avaliações pós-operatórias os parâmetros que mais melhoraram foram a dor corporal, a função física, a vitalidade e a função social ${ }^{(8)}$. Pelo contrário, a saúde mental e o desempenho emocional ainda não alcançavam valores normais aos 6 meses após cirurgia.

Uma revisão sistemática recente analisou 31 trabalhos sobre a QV após ATJ, concluindo que a ATJ proporciona uma melhor QV de forma precoce após o procedimento cirúrgico, essencialmente pela redução da dor e incremento da funcionalidade ${ }^{(9)}$. Os fatores do préoperatório que mais se correlacionaram positivamente com a QV no pós-operatório foram a menor claudicação, melhor qualidade do sono, os níveis de atividade física praticada antes do procedimento e suporte familiar e social adequado ${ }^{(9)}$.

Vários estudos foram realizados utilizando instrumentos específicos para avaliação da funcionalidade do joelho, designadamente o KOOS (Knee injury and osteoarthritis outome score). Um deles teve como participantes 39 pacientes submetidos a ATJ, os quais foram avaliados através de medidas de desempenho funcional, recorrendo designadamente ao teste de 6 minutos de marcha, Timed Up and Go e KOOS ${ }^{(10)}$. Os participantes foram avaliados antes da cirurgia e em três momentos subsequentes $(1,3$ e 6 meses após a ATJ). A investigação revelou existirem piores resultados no teste de 6 minutos de marcha e no Up and Go após um mês, tendo os resultados melhorado posteriormente de forma significativa aos 3 e 6 meses. Quanto aos valores do KOOS, a investigação concluiu existir incremento na funcionalidade do joelho, em todos os momentos do seguimento, à exceção da subescala relacionada com as atividades desportivas e de lazer, cuja melhoria só foi notória a partir dos 3 meses ${ }^{(10)}$. Os autores mencionam que as medidas auto referidas são demasiado valorizadas pelos utentes aos 30 dias, provavelmente devido a melhorias no alívio da dor, mas que tal não se reflete no desempenho funcional avaliado pelos 6 minutos de marcha e teste Timed Up and Go ${ }^{(10)}$.

Tendo em conta o anteriormente referido desenvolvemos uma investigação que teve como objetivo geral avaliar a perceção da funcionalidade/ problemas no joelho e a QVRS em pessoas idosas submetidos a artroplastia total do joelho. Constituíram nossos objetivos específicos. (i) Caracterizar os pacientes segundo as variáveis sociodemográficas (sexo, idade) e índice de massa corporal (IMC); (ii) Comparar os resultados obtidos no período préoperatório e oito semanas após a cirurgia relativamente à perceção da qualidade de vida dos pacientes (iii) 
Comparar os resultados obtidos no período préoperatório e oito semanas após a cirurgia relativamente à perceção do grau de funcionalidade do joelho.

\section{MÉTODO}

Tendo em conta os objetivos da investigação desenhouse um estudo de natureza quantitativa e de caráter longitudinal prospetivo, com dois momentos de avaliação: antes da cirurgia e 8 semanas após o procedimento cirúrgico.

A amostra foi composta por todas as pessoas idosas com diagnóstico de OA do joelho que consecutivamente foram submetidos a ATJ no HTQ (Hospital Terra Quente) durante um período de 6 meses. Foram excluídos do estudo pacientes para revisão de prótese de joelho, pacientes submetidos a prótese parcial do joelho, pacientes que fizeram ATJ cuja patologia de base não fosse a OA do joelho e pacientes que tiveram as seguintes complicações pós-operatórias: infeção, deiscência da ferida cirúrgica e derrame articular. De referir que todos os pacientes que participaram no estudo tiveram, durante o internamento, cuidados de enfermagem de reabilitação, designadamente ao nível do posicionamento do membro intervencionado, primeiro levante do leito, recuperação da amplitude articular e ensino e preparação para a alta.

Para além das variáveis sexo, idade e IMC, constituíram variáveis em estudo os seguintes constructos: (i) Qualidade de vida relacionada com a saúde, avaliada através do Medical Outcomes Study 36-Item Short-Form Health Survey (MOS SF-36 v2); (ii) Perceção da funcionalidade/problemas no joelho, avaliada através do Knee injury and osteoarthritis outome score (KOOS).

O MOS SF-36 v2 é utilizado para investigar a qualidade de vida de indivíduos com ou sem doença e encontra-se estruturado em 36 itens 0 seu tempo de preenchimento é em média de 10 minutos e pode ser preenchido pelo próprio ou por entrevista. As suas medidas de qualidade de vida são consideradas o padrão-ouro nos estudos relacionados à saúde ${ }^{(11)}$. O SF-36 v2 avalia a qualidade de vida na componente física e mental. A componente física integra as seguintes dimensões: Função física (FF), Desempenho físico (DF), Dor corporal (DC) e Saúde em geral (SG). Por sua vez a componente mental integra as seguintes dimensões: Vitalidade (VT), Função social (FS), Desempenho emocional (DE) e Saúde mental (SM).

Relativamente ao questionário KOOS este tem como objetivo medir a perceção da funcionalidade/ problemas no joelho. $O$ seu tempo de preenchimento médio é de 10 minutos e visa avaliar cinco dimensões que são pontuadas separadamente: dor ( 9 itens) outros sintomas para além da dor (7 itens), atividades de vida diária ( 7 itens), atividades desportivas e de lazer (5 itens) e qualidade de vida relacionada com a funcionalidade do joelho (4 itens). As pontuações por dimensão são apresentadas numa escala de orientação positiva em que 0 corresponde a problemas extremos e 100 corresponde a ausência de problemas no joelho.

Vários estudos mostram que o questionário KOOS apresenta boa evidência confiabilidade e validade de construto ${ }^{(12,13)}$.
O projeto do estudo foi aprovado pela administração do HTQ e todos os utentes aceitaram participar na investigação de forma livre e esclarecida, rubricando consentimento informado. Para evitar vieses os dados foram colhidos pelo mesmo investigador nos dois momentos de avaliação.

Para tratamento da informação recorreu-se ao programa estatístico SPSS (Statistical Packhage for the Social Sciences). Seguiram-se os procedimentos clássicos da estatística descritiva e inferencial. Utilizou-se o teste t para amostras emparelhadas para um nível de significância de referência $p<0,05$.

\section{RESULTADOS}

A amostra ficou constituída por 40 pessoas idosas, pertencendo na sua maioria ao sexo feminino $(62,5 \%)$. Encontrou-se uma média de idades próxima dos 72 anos e um IMC médio de $27,2 \mathrm{Kg} / \mathrm{m}^{2}$. 0 estado subjetivo de saúde foi avaliado pela primeira pergunta do SF-36 v2, questão que não integra qualquer dimensão do instrumento e é considerado um item de avaliação global da saúde autopercebida. Como se denota pela Tabela 1, verificou-se uma tendência mais favorável de respostas durante o segundo momento de avaliação (M2), onde $55 \%$ dos inquiridos referiu que a sua saúde era Boa, valor que compara com os $35 \%$ obtidos na primeira avaliação (M1). Por outro lado, observou-se um decréscimo entre $M 1$ e $M 2$ nas categorias saúde Razoável (52,5\% vs 37,5\%) e Fraca (7,5\% vs $2,5 \%)$.

\begin{tabular}{|l|c|c|}
\hline & \multicolumn{2}{|c|}{ Amostra } \\
\hline Género & \multicolumn{2}{|c|}{$25(62,5)$} \\
\hline Feminino, $n(\%)$ & \multicolumn{2}{|c|}{$15(37,5)$} \\
\hline Masculino, $n(\%)$ & \multicolumn{2}{|c|}{$40(100,0)$} \\
\hline TOTAL, $n(\%)$ & \multicolumn{2}{|c|}{$71,6 \pm 8,1$} \\
\hline Idade, $(M \pm D P)$ & \multicolumn{2}{|c|}{$27,2 \pm 3,9$} \\
\hline IMC, $(M \pm D P)$ & $1^{(2,5)}$ & $1^{(2,5)}$ \\
\hline $\begin{array}{l}\text { Em geral, diria que a } \\
\text { sua saúde é: }\end{array}$ & $1^{(2,5)}$ & $1^{(2,5)}$ \\
\hline Ótima, $n(\%)$ & $14(35,0)$ & $22(55,0)$ \\
\hline Muito boa, $n(\%)$ & $21^{(52,5)}$ & $15(37,5)$ \\
\hline Boa, $n(\%)$ & $3^{(7,5)}$ & $1^{(2,5)}$ \\
\hline Razoável & $40(100,0)$ & $40(100,0)$ \\
\hline Fraca, $n(\%)$ & \\
\hline TOTAL, $n(\%)$ &
\end{tabular}

Tabela 1- Participantes no estudo distribuídos por género, média de idades e valores médios de IMC. Resultados obtidos para o estado subjetivo de saúde

Na Tabela 2 comparam-se as médias obtidas na componente física do SF-36 v2 (FF, DF, DC e SG) nos dois momentos avaliados. Verificaram-se melhorias estatisticamente significativas $(p<0,05)$ em todas as dimensões da componente física ao segundo momento de avaliação. 


\begin{tabular}{|l|c|c|c|}
\hline & Momento 1 & Momento 2 & \multirow{2}{*}{$p$} \\
\cline { 2 - 3 } & $(M \pm D P)$ & $(M \pm D P)$ & \\
\hline Função física (FF) & $48,8 \pm 7,0$ & $53,6 \pm 11,1$ & 0,005 \\
\hline Desempenho físico (DF) & $52,5 \pm 11,0$ & $58,4 \pm 11,2$ & 0,001 \\
\hline Dor corporal (DC) & $42,2 \pm 12,4$ & $71,6 \pm 12,0$ & $<0,001$ \\
\hline Saúde em geral (SG) & $61,9 \pm 11,2$ & $67,8 \pm 10,0$ & $<0,001$ \\
\hline
\end{tabular}

M- Média; DP- Desvio padrão; p- Significância teste t para amostras emparelhadas

Tabela 2- Médias obtidas nos dois momentos de avaliação para as dimensões Função física, Desempenho físico, Dor corporal e Saúde em geral

Relativamente à componente mental do SF-36 v2, e analisando as variações nas médias registadas em M1 e M2 nas diferentes subescalas (VT, FS, DE, SM) através do teste $t$ para amostras emparelhadas (Tabela 3 ), concluímos por uma melhoria significativa $(p<0,05)$ na qualidade de vida em todas as dimensões, à exceção da vitalidade $(\mathrm{VT})(\mathrm{p}=0,062)$.

\begin{tabular}{|l|c|c|c|}
\hline & Momento 1 & Momento 2 & \multirow{2}{*}{$p$} \\
\cline { 2 - 3 } & $(M \pm D P)$ & $(M \pm D P)$ & \\
\hline Vitalidade (VT) & $58,9 \pm 7,2$ & $62,0 \pm 8,3$ & 0,062 \\
\hline Função social (FS) & $54,3 \pm 13,4$ & $69,2 \pm 9,7$ & $<0,001$ \\
\hline $\begin{array}{l}\text { Desempenho } \\
\text { emocional (DE) }\end{array}$ & $54,5 \pm 14,0$ & $66,5 \pm 12,6$ & $<0,001$ \\
\hline Saúde mental (SM) & $55,3 \pm 11,0$ & $64,0 \pm 7,5$ & $<0,001$ \\
\hline
\end{tabular}

M- Média; DP- Desvio padrão; p- Significância teste t para amostras emparelhadas

Tabela 3- Médias obtidas nos dois momentos de avaliação para as dimensões Vitalidade, Função social, Desempenho emocional e Saúde mental

A comparação entre os valores médios da primeira e segunda avaliação relativa ao questionário KOOS (Tabela 4) revela significância estatística nas dimensões sintomas, dor e qualidade de vida. Já a diferença de médias observadas para as atividades de vida diária e desporto/atividades de lazer não mostraram ter relevância estatística.

\begin{tabular}{|c|c|c|c|}
\hline & Momento 1 & Momento 2 & \multirow{2}{*}{$p$} \\
\cline { 2 - 3 } & $(M \pm D P)$ & $(M \pm D P)$ & \\
\hline Sintomas & $62,4 \pm 15,4$ & $66,5 \pm 11,9$ & 0,015 \\
\hline Dor & $58,6 \pm 12,5$ & $71,8 \pm 15,6$ & $<0,001$ \\
\hline $\begin{array}{c}\text { Atividades de vida } \\
\text { diária }\end{array}$ & $61,7 \pm 13,2$ & $64,0 \pm 11,1$ & 0,060 \\
\hline $\begin{array}{c}\text { Desporto/ } \\
\text { atividades de lazer }\end{array}$ & $13,6 \pm 3,0$ & $13,9 \pm 11,4$ & 0,885 \\
\hline Qualidade de vida & $43,8 \pm 11,4$ & $49,9 \pm 11,4$ & $<0,001$ \\
\hline
\end{tabular}

M- Média; DP- Desvio padrão; p- Significância teste t para amostras emparelhadas

Tabela 4 - Comparação das médias obtidas nos dois momentos de avaliação no questionário KOOS

\section{DISCUSSÃO}

O primeiro tópico de discussão refere-se ao facto da amostra estudada ser constituída maioritariamente por elementos do sexo feminino. Em termos epidemiológicos este resultado é corroborado por estudos que encontraram uma maior prevalência de OA nas mulheres, designadamente $72 \%{ }^{(14)}$ e $88,5 \%{ }^{(15)}$. Estes resultados poderão explicar-se pela osteoporose pósmenopáusica e pelo facto das mulheres apresentarem menor quantidade de massa muscular que os homens $(16,17)$.

Relativamente ao IMC foi obtido um valor médio de $27,2 \mathrm{Kg} / \mathrm{m} 2$. O excesso de peso, pelo aumento da pressão intra-articular que provoca, é considerado um fator de risco acrescido para o desenvolvimento de OA do joelho. Os resultados do estudo Framingham evidenciam que as mulheres que perderam cerca de 5 $\mathrm{kg}$ do seu peso apresentaram uma redução de $50 \%$ no risco de desenvolvimento de OA sintomática do joelho (18). Por outro lado, a perda de peso ponderal foi associada a melhoria da função física e redução da dor em múltiplos estudos ${ }^{(19,20)}$.

Quanto ao SF-36 v2, obtivemos mudanças significativas na direção da melhoria do estado de saúde e QV, ao segundo momento de avaliação, relativamente a todas as dimensões da componente física, e a todas as dimensões da componente mental, à exceção da vitalidade. Fracasso e Kaipper (2012) estudaram a perceção de funcionalidade em atividades de vida diária e qualidade de vida em pacientes submetidos a ATJ e verificaram, através da aplicação do MOS SF-36 que houve melhoria na sintomatologia, bem como nas limitações funcionais com tendência à melhoria da qualidade de vida após 15 dias da cirurgia (21). Uma revisão sistemática concluiu que a ATJ melhora a qualidade de vida especialmente no que diz respeito à dor e à funcionalidade ${ }^{(9)}$.

No nosso estudo a análise da perceção da funcionalidade/problemas no joelho foi realizada através do KOOS, concluindo-se por uma melhoria significativa nos sintomas, na dor e na qualidade de vida. Os nossos resultados vão de encontro aos reportados por um estudo que avaliou 13 pacientes no pré-operatório e às 4 semanas após cirurgia, concluindo por uma diminuição significativa da dor nesse período de tempo (22). Stevens-Lapsley e colaboradores desenvolveram um estudo com 39 pacientes submetidos a ATJ, avaliados em três momentos distintos $(1,3$ e 6 meses após a cirurgia) através de medidas de desempenho funcional, designadamente recorrendo ao teste de 6 minutos de marcha, Timed Up and Go e questionário KOOS ${ }^{(10)}$. Quanto aos resultados do KOOS, a investigação concluiu existir incremento na funcionalidade do joelho, em todos os momentos do seguimento, à exceção da subescala relacionada com as atividades desportivas e de lazer, cuja melhoria só foi notória a partir da avaliação aos 3 meses ${ }^{(10)}$.

\section{CONCLUSÃO}

Relativamente à qualidade de vida avaliada pelo MOS SF-36 v2 concluiu-se haver melhorias estatisticamente 
significativas $(p<0,05)$ em todas as dimensões da componente física (função física, desempenho físico, dor corporal e saúde em geral) ao segundo momento de avaliação. Na componente mental houve melhoria ( $p$ $<0,05$ ) em todas as dimensões (função social, desempenho emocional e saúde mental), à exceção da vitalidade $(p=0,062)$.

Para as dimensões do questionário utlizado para avaliação a perceção da funcionalidade/problemas do joelho (KOOS), verificou-se que na segunda avaliação houve uma tendência para diminuição da dor e melhoria dos sintomas.

Tendo em conta que o conceito de qualidade de vida se relaciona com o estado de saúde da pessoa e que a dor e a limitação funcional provocada pela OA influenciam negativamente 0 bem-estar e a realização das atividades de vida diária, pode concluir-se que a ATJ contribui para a melhoria da qualidade e desempenho físico da pessoa. Observou-se ainda que a ATJ além de revelar melhoria no desempenho físico, contribuiu também de forma bastante significativa para a melhoria da atividade emocional e social dos participantes.

O nosso estudo apresenta várias limitações das quais salientamos as decorrentes de um processo de amostragem não-probabilística com um pequeno número de participantes, o que obstaculiza a generalização dos resultados. Contudo, os dados que apresentamos poderão servir como valores comparativos em programas de reabilitação em pacientes submetidos a ATJ. Por outro lado, e dado o pequeno número de estudos realizados em Portugal que recorreram ao questionário KOOS, o presente estudo poderá incentivar o uso desta ferramenta na avaliação da funcionalidade do joelho, ao nível da prática especializada em enfermagem de reabilitação. Sugerese a realização de novos estudos com amostras mais extensas e com diferentes intervalos temporais de follow-up. Sugere-se ainda, em estudos futuros, a inclusão de outras variáveis relacionadas com o processo de reabilitação, designadamente se o mesmo teve continuidade após a alta da unidade hospitalar.

\section{REFERÊNCIAS BIBLIOGRÁFICAS}

1. Litwic A, Edwards MH, Dennison EM, Cooper C. Epidemiology and burden of osteoarthritis. Br Med Bull. 2013;105:185-99.

2. Plotnikoff R, Karunamuni N, Lytvyak E, Penfold C, Schopflocher $\mathrm{D}$, Imayama I, et al. Osteoarthritis prevalence and modifiable factors: a population study. BMC Public Health. 2015;15:1195.

3. Loeser RF. Age-related changes in the musculoskeletal system and the development of osteoarthritis. Clin Geriatr Med. $2010 ; 26^{(3)}: 371-86$.

4. Pal CP, Singh P, Chaturvedi S, Pruthi KK, Vij A. Epidemiology of knee osteoarthritis in India and related factors. Indian J Orthop. $2016 ; 50^{(5)}: 518-22$.

5. Farr li J, Miller LE, Block JE. Quality of life in patients with knee osteoarthritis: a commentary on nonsurgical and surgical treatments. Open Orthop J. 2013;7:619-23.

6. Preto LSR, Cruz FC, Mendes ME, Novo AFM. Qualidade de vida e saúde de pessoas submetidas a cirurgia de descompressão do nervo mediano. Revista de Enfermagem Referência. 2015; IV ${ }^{(5)}: 101-8$.

7. Heidari B. Knee osteoarthritis diagnosis, treatment and associated factors of progression: part II. Caspian J Intern Med.
$2011 ; 2^{(3)}: 249-55$.

8. Tsonga T, Kapetanakis S, Papadopoulos C, Papathanasiou J, Mourgias N, Georgiou N, et al. Evaluation of improvement in quality of life and physical activity after total knee arthroplasty in greek elderly women. Open Orthop J. 2011;5:343-7.

9. Silva RR, Santos AA, Júnior JDS, Matos MA. Quality of life after total knee arthroplasty: systematic review. Rev Bras Ortop. $2014 ; 49^{(5)}: 520-7$

10. Stevens-Lapsley JE, Schenkman ML, Dayton MR. Comparison of self-reported knee injury and osteoarthritis outcome score to performance measures in patients after total knee arthroplasty. PM\&R. 2011; $3^{(6)}: 541-9$.

11. Matcham F, Norton S, Steer S, Hotopf M. Usefulness of the SF-36 Health Survey in screening for depressive and anxiety disorders in rheumatoid arthritis. BMC Musculoskelet Disord. 2016;17 ${ }^{(1)}: 1$ 10.

12. Garratt AM, Brealey S, Gillespie WJ, Team DT. Patient-assessed health instruments for the knee: a structured review. Rheumatology (Oxford). 2004;43 ${ }^{(11)}: 1414-23$.

13. Roos EM, Lohmander LS. The Knee injury and Osteoarthritis Outcome Score (KOOS): from joint injury to osteoarthritis. Health Qual Life Outcomes. 2003;1:64.

14. Pires R, Albuquerque VG, Sturm L. Análise da reprodutibilidade de três classificações para a osteoartrose do joelho. Revista Brasileira de Ortopedia. 2008;43(8):329-35.

15. Vasconcelos K, Dias J, Dias R. Relação entre intensidade de dor e capacidade funcional em indivíduos obesos com osteoartrite do joelho. Revista Brasileira de Fisioterapia. 2006;10(2):213-8.

16. Preto LSR, Conceição MDC, Figueiredo TM, Mata MAP, Preto PMB, Mateo Aguilar E. Frailty, body composition and nutritional status in non-institutionalised elderly. Enferm Clin. 2017; 27(6):339345.

17. Novo AFM, Mendes ME, Preto LSR, Azevedo A, Vaz J, Correia J, editors. Physical capacity, quality of life and body composition of postmenopausal osteoporotic women. 7th EFSMA-European Congress of Sports Medicine 3rd Central European Congress of Physical Medicine and Rehabilitation; 2011; Salzburg: Austrian Society of Physical Medicine and Rehabilitation.

18. Allen K, Golightlya Y. Epidemiology of osteoarthritis: state of the evidence. Curr Opin Rheumatol. 2015;27(3):276-83.

19. Messier SP, Loeser RF, Miller GD, Morgan TM, Rejeski WJ, Sevick $M A$, et al. Exercise and dietary weight loss in overweight and obese older adults with knee osteoarthritis: the Arthritis, Diet, and Activity Promotion Trial. Arthritis Rheum. 2004;50(5):150110.

20. Christensen R, Bartels EM, Astrup A, Bliddal H. Effect of weight reduction in obese patients diagnosed with knee osteoarthritis: a systematic review and meta-analysis. Ann Rheum Dis. $2007 ; 66^{(4)}: 433-9$

21. Fracasso BV, Kaipper MB. Avaliação da funcionalidade em indivíduos submetidos à artroplastia total do joelho. Cippus. $2012 ; 1^{(2)}: 170-84$

22. Wickham-Bruno R. Assessment of strength, balance, and function before and after total knee arthroplasty: Ball State University. 2008. 